\title{
Autophagy regulates proliferation and biliary differentiation of hepatic oval cells via the MAPK/ERK signaling pathway
}

\author{
DONGDONG CHEN $^{1 *}$, XIAOXIAO WU ${ }^{2 *}$, JIE ZHENG $^{1}$, RUIJIE DAI $^{1}$, ZHICHAO MO $^{1}$, \\ FAHAD MUNIR $^{1}$, XIAOLONG NI $^{1}$ and YUNFENG SHAN ${ }^{1}$ \\ Departments of ${ }^{1}$ Hepatobiliary Surgery and ${ }^{2}$ Intensive Care Unit, The First Affiliated Hospital of \\ Wenzhou Medical University, Wenzhou, Zhejiang 325000, P.R. China
}

Received November 7, 2016; Accepted July 7, 2017

DOI: $10.3892 / \mathrm{mmr} .2017 .8157$

\begin{abstract}
Hepatic oval cells (HOCs) are thought to possess self-renewal ability and a bipotential capacity for differentiation, which allows them to differentiate into hepatocytes and cholangiocytes. Autophagy serves an important role in self-renewal and differentiation of stem cells; however, how autophagy contributes to proliferation and differentiation of hepatic progenitor cells has yet to be elucidated. In the present study, autophagy was regulated by rapamycin (Rapa) and chloroquine (Chlo) administration. The results demonstrated that Chlo-treated HOCs exhibited decreased autophagic activity alongside a decreased tendency to proliferate, as determined by Cell Counting Kit-8. In addition, activation of autophagy by Rapa enhanced the biliary differentiation of HOCs. Furthermore, increased phosphorylated (p)-extracellular signal-regulated kinase (ERK)/p-p38 expression was observed following the induction of autophagy, thus indicating that the mitogen-activated protein kinase (MAPK)/ERK signaling pathway was activated by autophagy to exert effects on the stimulation of HOC proliferation and differentiation. In conclusion, the present study demonstrated that autophagy regulates proliferation and biliary differentiation of HOCs via the MAPK/ERK signaling pathway. These results suggest a role for autophagy in stimulating the proliferation and differentiation of HOCs.
\end{abstract}

Correspondence to: Professor Yunfeng Shan, Department of Hepatobiliary Surgery, The First Affiliated Hospital of Wenzhou Medical University, 2 Qianan Road, Nanbaixiang Street, Ouhai, Wenzhou, Zhejiang 325000, P.R. China

E-mail: shanyf@yahoo.com

*Contributed equally

Abbreviations: CK19, cytokeratin 19; HOC, hepatic oval cell; Rapa, rapamycin; Chlo, chloroquine; SB, sodium butyrate; MAPK, mitogen-activated protein kinase

Key words: hepatic oval cells, autophagy, proliferation, differentiation, MAPK/extracellular signal-regulated kinase signaling

\section{Introduction}

Hepatic oval cells (HOCs) are thought to possess self-renewal ability and a bipotential capacity for differentiation, which allows them to differentiate into hepatocytes and cholangiocytes (1). The differentiation of HOCs has been reported to be regulated by various signaling pathways, including Notch, Wnt, transforming growth factor- $\beta$, bone morphogenetic protein, hepatocyte growth factor and fibroblast growth factor signaling pathways (2).

Macroautophagy, which is also referred to as autophagy, is a highly conserved and important metabolic process in eukaryotic cells. Autophagy has been characterized as an essential process associated with cellular homeostasis. Under stress or starvation conditions, unnecessary or dysfunctional cellular components are degraded and recycled through autophagy, thus promoting cell survival and growth (3). Autophagy, which is initiated by the microenvironment or cytokines, serves a role in various physiological and pathological processes, including cancer, metabolic disease, neurodegenerative disorders, cell growth, cell death, ontogeny, and cell differentiation and self-renewal (4). However, how autophagy contributes to proliferation and differentiation of HOCs remains to be elucidated.

Mitogen-activated protein kinases (MAPKs) are a family of serine/threonine kinases, which function as broad intracellular signal mediators of cell survival, proliferation, motility, apoptosis and differentiation (5). In addition, MAPKs are essential for stem cell self-renewal and commitment to lineage differentiation (6). Extracellular signal-regulated kinase (ERK) $1 / 2$ is required for osteogenic mesenchymal stem cell differentiation and skeletal development (7). It has previously been reported that MAPK/ERK serves an important role in the proliferation of HOCs (8). Furthermore, induction of autophagy is associated with epidermal growth factor-induced MAPK phosphorylation. However, the effects of autophagy on the MAPK/ERK signaling pathways in HOC proliferation and biliary differentiation are currently not well understood. Therefore, to investigate these effects, as well as the underlying molecular mechanisms, the present study investigated the effects of autophagy on HOC proliferation, apoptosis and differentiation into cholangiocytes, and the role of the MAPK/ERK signaling pathway in these processes. 


\section{Materials and methods}

Cell lines, culture and drug treatment. The WB-F344 HOC line was purchased from the Cell Bank of the Type Culture Collection of the Chinese Academy of Sciences (Shanghai, China). This cell line possesses stem cell features and has been reported previously (9). WB-F344 cells were cultured in a $25 \mathrm{~cm}^{3}$ flask with RPMI-1640 medium, supplemented with $10 \%$ (v/v) fetal bovine serum (FBS) (both from (Gibco; Thermo Fisher Scientific, Inc., Waltham, MA, USA), $100 \mu \mathrm{g} / \mathrm{ml}$ streptomycin (Sigma-Aldrich; Merck KGaA, Darmstadt, Germany) and $100 \mathrm{U} / \mathrm{ml}$ penicillin (Gibco; Thermo Fisher Scientific, Inc.) in a humidified incubator containing $5 \% \mathrm{CO}_{2}$ at $37^{\circ} \mathrm{C}$. The culture medium was changed every second day and cells were passaged $1: 3$ using $0.05 \%$ trypsin plus $0.02 \%$ EDTA. To regulate autophagy in WB-F344 cells, chloroquine (Chlo; $100 \mathrm{mg}$ ) and rapamycin (Rapa; $1.0 \mathrm{mg} / \mathrm{l}$ ) (both from Sigma-Aldrich; Merck KGaA, Darmstadt, Germany) were administered to inhibit or induce autophagy, respectively (10). Briefly, WB-F344 cells were seeded in 6-well culture plates at $3 \times 10^{5}$ cells/well and were cultured overnight. The following day, the cells were treated with or without Rapa $(40 \mathrm{nM})$ or Chlo $(50 \mu \mathrm{M})$ for $24 \mathrm{~h}$ at $37^{\circ} \mathrm{C}$. For the following experiments, Rapa and Chlo were dissolved in dimethyl sulfoxide (DMSO; Sigma-Aldrich; Merck KGaA). Control cells were treated with an equal amount of DMSO used for drug treatment [not exceeding $0.1 \%(\mathrm{v} / \mathrm{v})]$. Morphology of WB-F344 cells was evaluated by a light microscope (Olympus CX21; Olympus Corporation, Tokyo, Japan) after Rapa or Chlo treatment.

Biliary differentiation of hepatic progenitor cells. WB-F344 cells had been used as a good model to study the mechanism of biliary differentiation (11). To induce biliary differentiation, WB-F344 cells which were seeded in 6-well culture plates at a density of $5 \times 10^{5}$ cells/well were treated with $3.75 \mathrm{mM}$ sodium butyrate (SB; Sigma-Aldrich; Merck KGaA) (11). The medium was changed every 2 days. The days of differentiation were numbered consecutively beginning at the first day of the SB treatment ( 0 day) to the last day ( 3 day).

Determination of cell viability. Cell viability was detected using the Cell Counting Kit (CCK)-8 assay. WB-F344 cells were plated in 96-well plates with three duplicate wells in each group. When the cells had grown to $70-80 \%$ confluency, the cells were treated with or without Rapa (30 and $700 \mathrm{nM}$ ) or Chlo $(20-500 \mu \mathrm{M})$. The CCK-8 solution $(10 \mu \mathrm{l})$ at a 1:10 dilution in FBS-free RPMI-1640 medium (100 $\mu$ l) was added to each well, and the plates were incubated for $2 \mathrm{~h}$ at $37^{\circ} \mathrm{C}$. Absorbance was measured at $450 \mathrm{~nm}$ using a microplate reader (Molecular Devices, LLC, Sunnyvale, CA, USA). The mean optical density (OD) of three wells in the indicated groups was used to calculate the inhibition rate of the cells as follows: Inhibition rate $=\left(\mathrm{OD}_{\text {control group }}-\mathrm{OD}_{\text {treatment group }}\right) /\left(\mathrm{OD}_{\text {control group }}-\mathrm{OD}_{\text {blank }}\right.$ group $\mathrm{x} 100 \%$. The experiment was performed in quadruplicate.

Immunofluorescence staining. After treatment, $3 \times 10^{6}$ cells in 6-well plates, were fixed with $4 \%$ paraformaldehyde and incubated with CK19 (cat. no. 3092, 1:200; Cell Signaling Technology, Leiden, the Netherlands) overnight at $4^{\circ} \mathrm{C}$. And then incubated with Alexa Fluor 488-labeled secondary antibodies (cat. no. A-11034, 1:200; Molecular Probes; Thermo Fisher Scientific, Inc.) for $1 \mathrm{~h}$ at $37^{\circ} \mathrm{C}$. DAPI (Sigma-Aldrich; Merck $\mathrm{KGaA}$ ) was used to stain the nuclei. Five fields from each well were taken at magnification, x200. Fluorescence intensity was evaluated by using a confocal microscope (Leica TCS SP2; Leica Microsystems, GmbH, Wetzlar, Germany).

Flow cytometry to determine the effects of autophagy on apoptosis. To analyze cell cycle progression and to assess apoptosis, flow cytometric analysis was used to identify sub- $\mathrm{G}_{1}$ cells/apoptotic cells and to measure the percentage of sub- $\mathrm{G}_{1}$ cells after propidium iodide (PI) staining in hypotonic buffer as previously described (12). The cells were collected at $24 \mathrm{~h}$ after drug treatment and apoptotic cells were analyzed by flow cytometry using a fluorescein isothiocyanate (FITC)-Annexin V Apoptosis Detection kit (cat. no. 556547; BD Biosciences, Franklin Lakes, NJ, USA). Briefly, following trypsinization and three washes in PBS, cells were resuspended in $500 \mu \mathrm{l} 1 \mathrm{X}$ Annexin V binding buffer, $5 \mu \mathrm{l}$ FITC-Annexin $\mathrm{V}$ and $5 \mu \mathrm{P}$ PI, and were incubated in the dark for $15 \mathrm{~min}$ at $37^{\circ} \mathrm{C}$ after gentle mixing. Following the addition of $200 \mu 11 \mathrm{X}$ Annexin $\mathrm{V}$ binding buffer, apoptosis was analyzed by flow cytometry (BD FACSCanto II; BD Biosciences, San Jose, CA, USA) within $1 \mathrm{~h}$. For cell cycle analysis after $24 \mathrm{~h}$, after drug treatment cells were washed with cold PBS for three times and fixed in $70 \%$ ethyl alcohol at $4^{\circ} \mathrm{C}$ overnight. Cells were then treated with $10 \mu \mathrm{g} / \mathrm{ml}$ RNase and were stained with $50 \mu \mathrm{g} / \mathrm{ml}$ PI for $30 \mathrm{~min}$ at room temperature in the dark. The cell cycle was then measured by BD FACSCanto II and the cell cycle distribution was analyzed by ModFit LT v3.3 software (BD Biosciences).

Western blot analysis. Western blot analysis was performed as described previously (12). Briefly, following cell lysis and protein extraction with radioimmunoprecipitation assay lysis buffer (Beyotime Institute of Biotechnology, Haimen, China), protein concentration was measured using the bicinchoninic acid protein assay reagent. The samples $(50 \mu \mathrm{g})$ were then denatured in sample buffer which containing SDS and the proteins were separated by 10-12\% SDS-PAGE gels. The proteins were then electroblotted onto polyvinylidene difluoride membranes. After blocking with 5\% skimmed milk for $1 \mathrm{~h}$ at room temperature, the blots were incubated with rabbit anti-microtubule-associated proteins $1 \mathrm{~A} / 1 \mathrm{~B}$ light chain $3 \mathrm{~B}$ (LC3B; cat. no. 4108, 1:1,000), rabbit anti-p62 (cat. no. 5114, 1:1,000), rabbit anti-cytokeratin 19 (CK19; cat. no. 13092, 1:1,000), and rabbit anti-cleaved PARP (cat. no. 9545, 1:1,000) (all from CST Biological Reagents Co., Ltd., Shanghai, China), rabbit anti-Bcl-2 (cat. no. ab59348, 1:1,000) or anti-GAPDH (CST Biological Reagents CO., Ltd., cat. no. 5174, 1:1,000) antibodies $(1: 2,000)$ overnight at $4^{\circ} \mathrm{C}$. Membranes were also incubated with anti-phosphorylated (p)-p38 MAPK (Thr180/Tyr182; cat. no. 9215, 1:1,000) and p-p44/42 MAPK (ERK1/2; cat. no. 41370, 1:1,000) (both from CST Biological Reagents Co., Ltd.) antibodies (Cell Signaling Technology, Inc., Danvers, MA, USA) at a 1:1,000 dilutions. Blots were stripped and re-probed with anti-p38 MAPK (cat. no. 92121; Abcam, Cambridge, MA, USA) and anti-p44/42 MAPK (ERK1/2; cat. no. 4695, 1:1,000; CST Biological Reagents Co., Ltd.) antibodies at a 1:2,000 dilution. After three washes with 
Tris-buffered saline containing $0.05 \%$ Tween-20, membranes were incubated with horseradish peroxidase-conjugated secondary antibodies (cat. no. GHPA002, 1:2,000; Xi'an Guanyu Bio-Tech Co., Ltd., Xi'an, China) for $1 \mathrm{~h}$ at room temperature. The membranes were washed a further three times for $5 \mathrm{~min}$. Blots were visualized using the Pierce Enhanced Chemiluminescence Western Blotting Substrate (Thermo Fisher Scientific, Inc.) followed by autoradiography. Grey value analysis was performed using Image Lab software (version 5.2.1; Bio-Rad Laboratories, Inc., Hercules, CA, USA). To ensure equal loading, membranes were probed with an anti-GAPDH antibody.

Statistical analysis. All experiments were repeated three times. Data are presented as the mean \pm standard error and were analyzed using SPSS 13.0 software (SPSS Inc., Chicago, IL, USA). Data were statistically analyzed by one-way analysis of variance followed by Dunnett's test for multiple comparisons. $\mathrm{P}<0.05$ was considered to indicate a statistically significant difference.

\section{Results}

Pharmacological regulation of autophagic activity in $W B-F 344$ cells. The present study detected the autophagic activity of WB-F344 cells following treatment with Chlo or Rapa for $24 \mathrm{~h}$. Treatment with Rapa, which is known to induce autophagy, for $24 \mathrm{~h}$ promoted the ratio of LC3A/BII and LC3A/BI and the degradation of p62/sequestome 1, which is a selective substrate of autophagy. Conversely, treatment with the autophagy inhibitor Chlo significantly enhanced LC3A/BII and p62 expression (Fig. 1A and B). These results indicated that Rapa was able to enhance autophagy, whereas Chlo inhibited autophagy in WB-F344 cells.

Inhibition of autophagy decreases WB-F344 cell proliferation and induces WB-F344 cell apoptosis. Proliferation of WB-F344 cells was measured 24 h after Rapa or Chlo treatment. As presented in Fig. 2A and B, Chlo significantly decreased the proliferation of WB-F344 cells $(\mathrm{P}<0.05)$. The anti-proliferative effects of Chlo on WB-F344 cells were verified using the CCK-8 assay, which can be used to examine cell proliferation. When the cells were treated with 20-500 $\mu \mathrm{mol} / 1$ Chlo for $24 \mathrm{~h}$ the proliferation of WB-F344 cells was inhibited in a time- and dose-dependent manner (Fig. 2B). However, Rapa, which induces autophagy, had no significant effect on viability (Fig. 2B). To determine whether cell cycle progression was affected by Rapa or Chlo treatment, a cell cycle analysis was conducted using flow cytometry. Rapa $(0.4 \% \pm 0.8)$ did not alter cell cycle progression, which is consistent with the findings of the CCK-8 assay. However, following treatment with $50 \mu \mathrm{M}$ Chlo $(2.8 \% \pm 1.2)$, subG $_{1}$ cell cycle arrest was increased compare with the control group $(0.5 \% \pm 0.9, \mathrm{P}<0.05$; Fig. $2 \mathrm{C})$. These findings indicated that Chlo induces apoptosis of WB-F344 cells. In order to confirm the apoptotic effects of Chlo, cellular apoptosis was determined by Annexin V-PI staining and flow cytometry. Flow cytometric analysis demonstrated that treatment with the autophagy inducer Rapa slightly reduced the apoptotic rate of WB-F344 cells $(3.4 \% \pm 0.6)$;
A
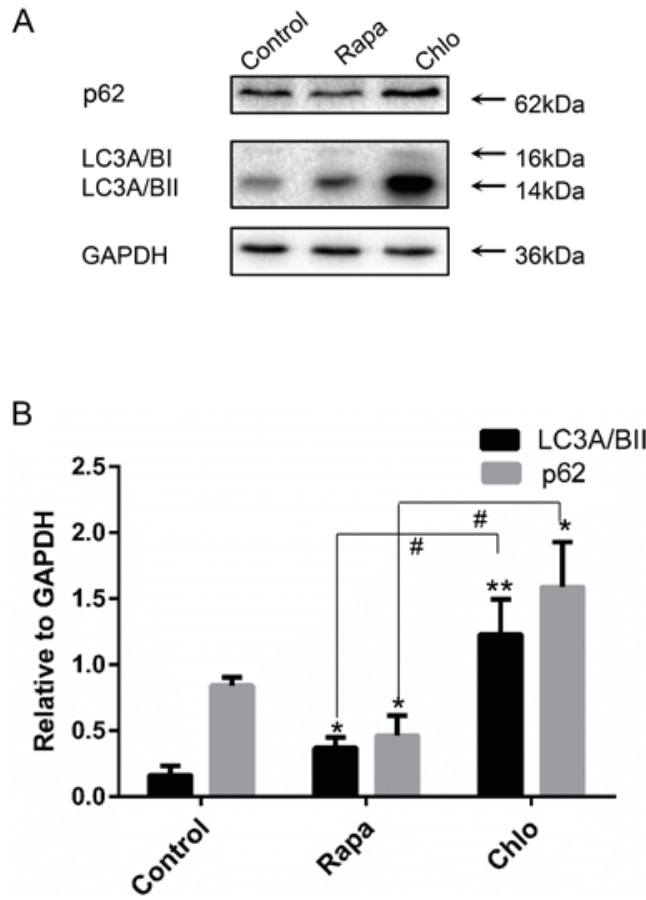

Figure 1. Effects of various drugs on autophagic activity of hepatic oval cells following $24 \mathrm{~h}$ incubation. (A) Cells were treated with various drugs for $24 \mathrm{~h}$; p62 and LC3 were detected by western blotting. (B) Graphical presentation of the relative abundance of LC3-II and p62 following normalization with GAPDH. Results are presented as the mean \pm standard error of the mean of four series of experiments. ${ }^{*} \mathrm{P}<0.05,{ }^{* *} \mathrm{P}<0.01$ vs. the control group; ${ }^{\#} \mathrm{P}<0.05$ Rapa vs. Chlo group. Chlo, chloroquine; LC3, microtubule-associated protein 1A/1B-light chain 3; Rapa, rapamycin.

however, this was not significantly different compared with the control group. $(5.4 \% \pm 0.7$; Fig. 2D). Following treatment with the autophagy inhibitor Chlo, the apoptotic rate of WB-F344 cells $(16.6 \% \pm 1.1)$ was significantly enhanced compared with the control group $(\mathrm{P}<0.05 ; 2 \mathrm{D})$. In Fig. $2 \mathrm{E}$ and $\mathrm{F}$, in the Rapa group, Rapa increased the expression of BCL-2 and decreased the expression of cleaved PARP $(\mathrm{P}<0.01)$. However, the results of chlo were the opposite of that of rapa $(\mathrm{P}<0.05)$. These results indicated that autophagy inhibits apoptosis, whereas inhibition of autophagy promotes apoptosis of WB-F344 cells. But in Fig. 2D. this was not significantly different compared with the control group. We believed that autophagy can reduce apoptosis, but autophagy can also promote cell death. Therefore, the apoptosis of Rapa group was not obvious compared with the control group.

Autophagy enhances the biliary differentiation of WB-F344 cells. Since autophagy can promote proliferation of WB-F344 cells, the present study aimed to determine whether autophagy exerts an effect on the differentiation of WB-F344 cells. Therefore, the effects of Rapa and Chlo were determined on the biliary differentiation of WB-F344 cells. WB-F344 cells were co-treated with sodium butyrate (SB) and Rapa or Chlo for 3 days. As presented in Fig. 3A, when WB-F344 cells were treated with SB, CK19 was slightly expressed in the cytoplasm; however, CK19 protein expression was significantly increased in the Rapa group compared with the control group $(\mathrm{P}<0.01)$. Furthermore, the results of the western blot analysis were consistent with the findings of immunofluorescence; upon 
A

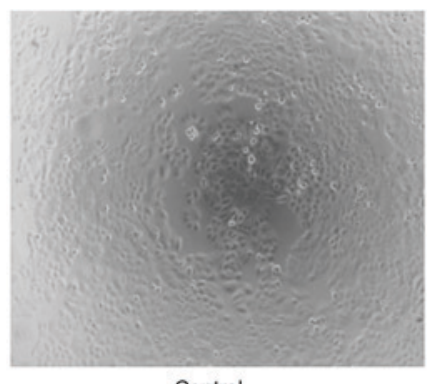

Control

B

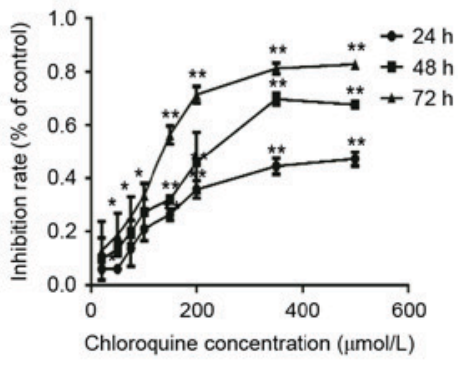

D

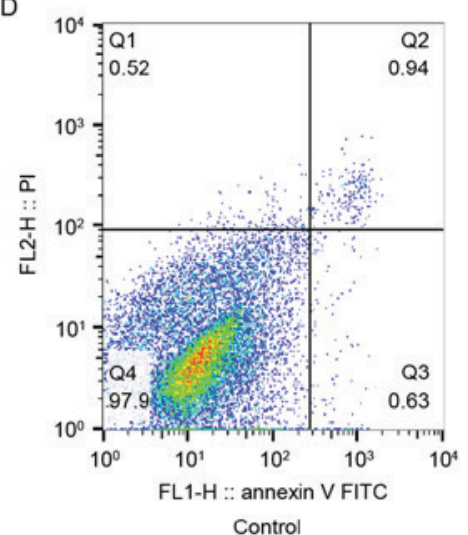

$E$

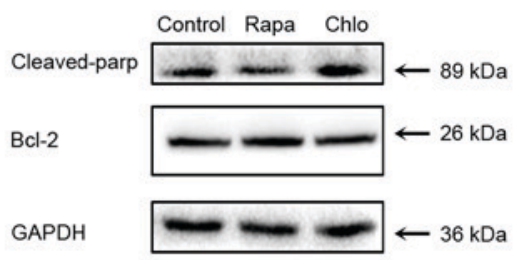

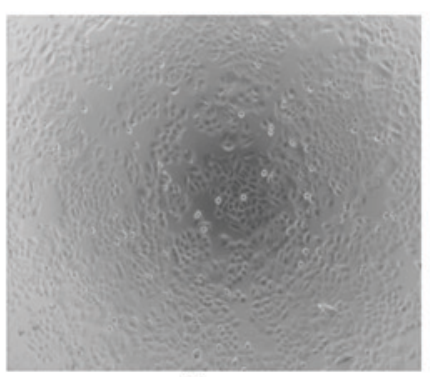

Rapa

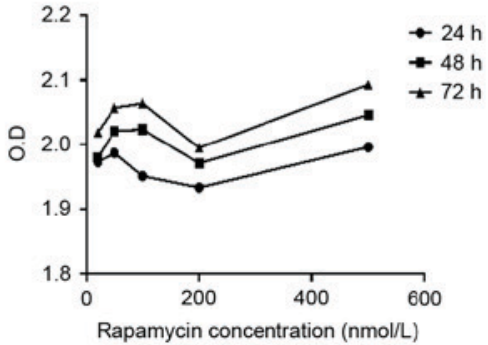

C
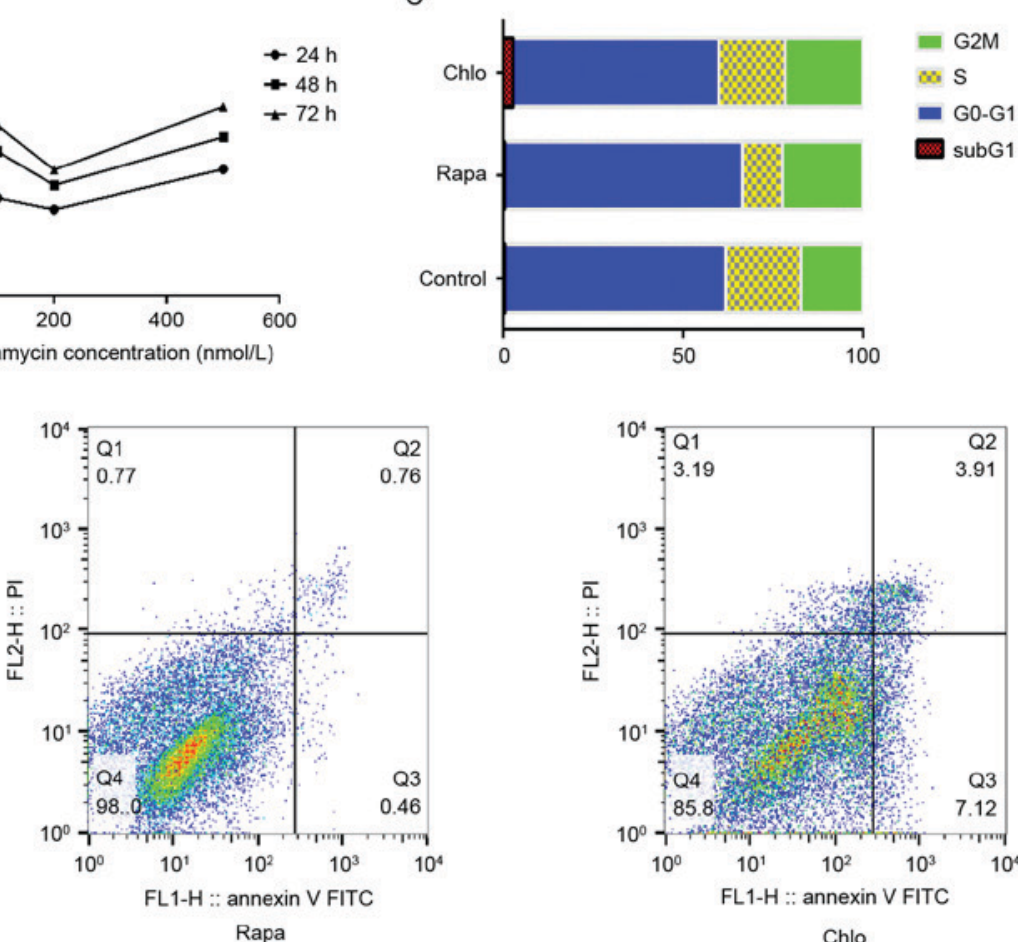

$\mathrm{F}$
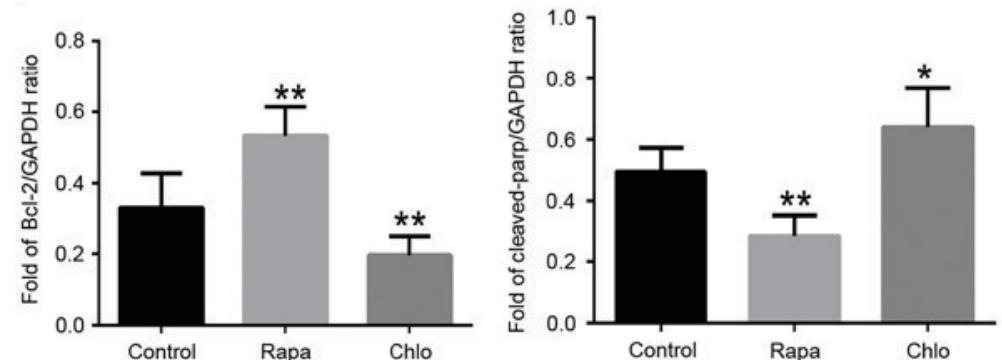

Figure 2. Chlo decreases the proliferation of WB-F344 cells and induces apoptosis. (A) Morphology of WB-F344 cells 24 h after Rapa or Chlo treatment (magnification, $\mathrm{x} 40)$. (B) Chlo $(20-500 \mu \mathrm{M})$ significantly decreased the proliferation of WB-F344 cells, as determined by Cell Counting Kit- 8 assay. Data are presented as the mean \pm standard deviation $(\mathrm{n}=3)$. ${ }^{*} \mathrm{P}<0.05,{ }^{* *} \mathrm{P}<0.01$ vs. the control group. (C) Cell cycle analysis indicated that $50 \mu \mathrm{M}$ Chlo increased cell cycle arrest at SubG1, which may induce apoptosis. (D) WB-F344 cells were analyzed by Annexin V and PI staining followed by flow cytometry. (E and F) Western blot analysis of the expression levels of cleaved PARP and Bcl-2 in WB-F344 cells. ${ }^{*} \mathrm{P}<0.05,{ }^{* *} \mathrm{P}<0.01$ vs. the control group. Bcl-2, B-cell lymphoma 2; Chlo, chloroquine; FITC, fluorescein isothiocyanate; OD, optical density; PARP, poly(ADP-ribose) polymerase; PI, propidium iodide; Rapa, rapamycin.

biliary differentiation, the expression of CK19 was increased in the Rapa-treated group compared with in the control group $(\mathrm{P}<0.01$; Fig. 3B and $\mathrm{C})$. These results indicated that activation of autophagy promotes the biliary differentiation of WB-F344 cells.

Effects of autophagy on the MAPK/ERK signaling pathway in WB-F344 cells. To determine the effects of MAPK/ERK signaling on autophagy-mediated enhancement of biliary differentiation of WB-F344 cells, western blotting was used to detect the expression levels of MAPK/ERK after modulation of autophagy. The results demonstrated that treatment with the autophagy inducer Rapa increased the phosphorylation of ERK1/2 and p38 (Fig. 4A) compared with in the control group. Semi-quantification of the blots indicated that the levels of p-ERK1/2 and p-p38 were increased 2 -fold 
A
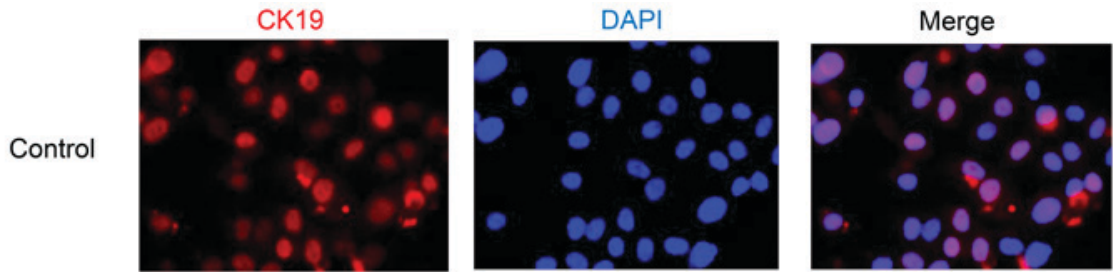

SB
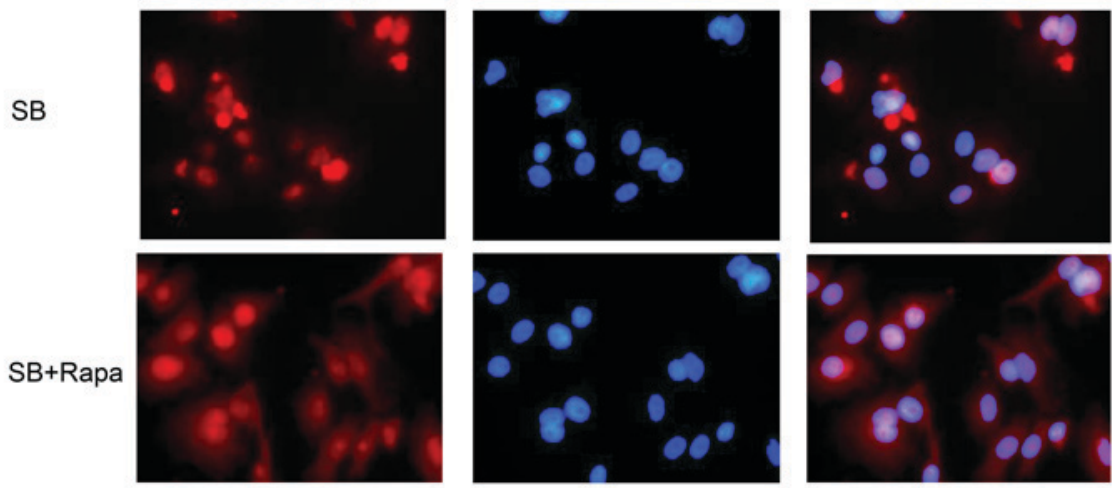

SB+Chlo
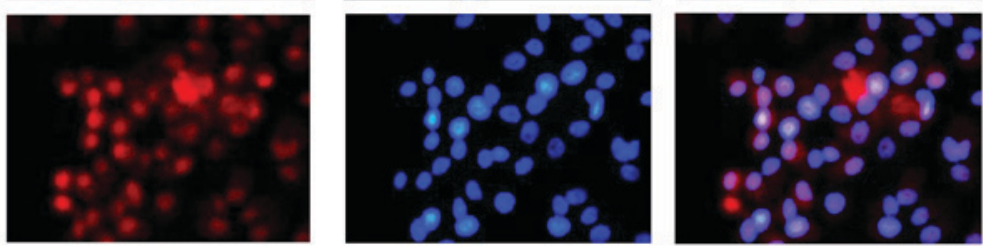

B

C
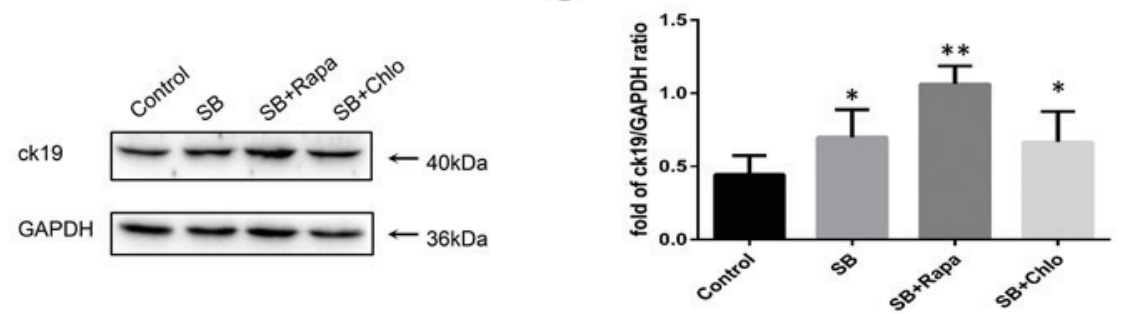

Figure 3. Effects of autophagy on differentiation of WB-F344 cells to bile duct epithelial cells in vitro. (A) Fluorescent immunocytochemistry of CK19 protein (red) and DAPI staining (blue) in WB-F344 cells (magnification, x200). (B) CK19 protein was detected by western blotting and was normalized to GAPDH. (C) Densitometric analysis of the protein bands (n=3/group). ${ }^{*} \mathrm{P}<0.05,{ }^{* * *} \mathrm{P}<0.01$ vs. the control group. Chlo, chloroquine; CK19, cytokeratin 19; Rapa, rapamycin; $\mathrm{SB}$, sodium butyrate.
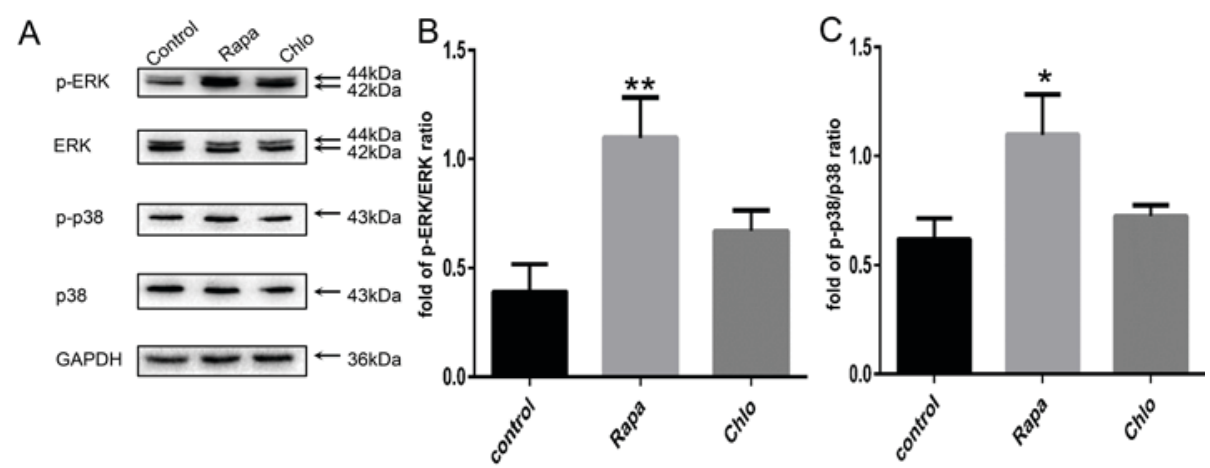

Figure 4. Effects of autophagy on the mitogen-activated protein kinase pathway in WB-F344 cells. Following incubation of WB-F344 cells with various drugs for $24 \mathrm{~h}$, (A) protein expression levels of p-ERK1/2, ERK1/2, p-p38 and p38 were determined by western blotting. (B and C) Ratio of p-ERK/ERK and p-p38/p38 intensity. Data are expressed as the mean \pm standard deviation. ${ }^{*} \mathrm{P}<0.05,{ }^{* *} \mathrm{P}<0.01$ vs. the control group. Chlo, chloroquine; ERK, extracellular signal-regulated protein kinase; p, phosphorylated; Rapa, rapamycin.

following Rapa treatment compared with the control group (Fig. 4B and C). However, the expression levels of p-ERK1/2 and p-p38 were not significantly altered in the Chlo group.
These results suggested that autophagy may promote differentiation of WB-F344 cells by upregulating the MAPK/ERK signaling pathway. 


\section{Discussion}

Autophagy has been demonstrated to protect cells from stress conditions, including hypoxia, and to serve an important role in self-renewal, proliferation, differentiation and apoptosis of stem cells (13-16). In addition, autophagy can efficiently and rapidly downregulate enzymes, transcription factors, adhesion molecules and certain secreted proteins $(17,18)$, which are important for stem cell proliferation and differentiation $(19,20)$. Autophagy mediates the degradation of cellular components, including proteins and organelles, in lysosomes (21), and contributes to cellular homeostasis, quality control and maintenance of energetic balance, which are essential for cell proliferation (15). In epidermal stem cells, mesenchymal stem cells, hematopoietic stem cells and dermal stem cells, basal autophagy levels are higher (22-26). After the induction of differentiation, autophagic levels are decreased. Nevertheless, autophagy is increased during the differentiation of embryonic stem cells, neural stem cells and cardiac stem cells (27-32). It has also been reported that autophagy serves a crucial role in regulating the stemness of hepatic progenitor cells (HPCs) (33). High autophagic levels are detected in the majority of the stem cells when they differentiate into other cells, which is essential for the maintenance of cellular stemness and homeostasis. There are many studies of the role of pathways on autophagy $(34,35)$. Therefore, the present study investigated the effects of autophagy on proliferation and differentiation of HOCs.

Stem cells undergo self-renewal and have the potential to differentiate into various cell populations. It has been established that a bipotential cell type, which has stem cell-like properties, exists in the adult liver and can differentiate into hepatic cells and cholangiocytes, these cells are referred to as HOCs or HPCs (36). HOCs are activated in reaction to various types of chronic liver disease (37).

It has been reported that autophagy regulates biliary differentiation of HPCs via the Notch1 signaling pathway (38). However, the role of autophagy in the proliferation, apoptosis and differentiation of WB-F344 cells remains to be elucidated. In the present study, Chlo was used to inhibit autophagy in WB-F344 cells, and was demonstrated to increase the SubG $_{1}$ population of cells and promote apoptosis, whereas treatment with Rapa exerted the opposite effects, thus suggesting that inhibition of autophagy inhibits WB-F344 cell proliferation. These results are consistent with previous studies, which reported that inhibition of autophagy suppresses proliferation and increases the population of apoptotic cells $(39,40)$. Although autophagy and apoptosis are mutually inhibitory (41), in the present study, autophagy appeared to exert anti-apoptotic effects.

The present study also aimed to determine the effects of autophagy on HOC-cholangiocyte differentiation, the results confirmed that SB induced WB-F344 cell differentiation into a cholangiocyte phenotype and revealed that the expression of the biliary marker, CK19, was significantly increased in response to Rapa-induced autophagy. These findings suggested that autophagy may be involved in WB-F344 cell differentiation. Conversely, a previous study by Zeng et al (38) demonstrated that activation of autophagy may suppress the biliary differentiation of WB-F344 cells.
Further investigation suggested that Rapa-induced activation of autophagy may stimulate the MAPK/ERK signaling pathway. These results are consistent with those of previous studies, which indicated that activation of the MAPK/ERK pathway can be induced by Rapa $(42,43)$, and may promote HOC proliferation (8). Therefore, the ERK signaling pathway may be considered an important regulator for the proliferation and differentiation of WB-F344 cells (8). These findings suggested that activation of MAPK/ERK signaling induced by autophagy is essential for cell differentiation.

HOCs are clinically significant, as they are potentially useful for cell and/or gene therapy for the treatment of metabolic liver diseases (37). However, the role of autophagy in WB-F344 cells involves alterations in signaling pathways, such as in Akt and Hedgehog, and the mechanisms are still highly heterogeneous $(34,35)$. The present study, demonstrated that inhibition of autophagy can inhibit HOCs proliferation and autophagy promoting HOCs differentiation. In addition, MAPK/ERK pathway is likely to be involved. Future studies are required to explore how autophagy affects the MAPK/ERK pathway during liver regeneration, and to reveal the potential mechanisms underlying HOC proliferation and differentiation, thus providing subclinical strategies for HOC-mediated liver regeneration or the inhibition of HOC-mediated tumorigenesis.

\section{Acknowledgements}

The present study was supported by grants from the Zhejiang Province Natural Science Foundation of China (grant no. LY12H03006).

\section{References}

1. Miyajima A, Tanaka M and Itoh T: Stem/progenitor cells in liver development, homeostasis, regeneration, and reprogramming. Cell Stem Cell 14: 561-574, 2014.

2. Mishra L, Banker T, Murray J, Byers S, Thenappan A, He AR, Shetty K, Johnson L and Reddy EP: Liver stem cells and hepatocellular carcinoma. Hepatology 49: 318-329, 2009.

3. Lapierre LR, Gelino S, Meléndez A and Hansen M: Autophagy and lipid metabolism coordinately modulate life span in germline-less C. elegans. Curr Biol 21: 1507-1514, 2011.

4. Choi AM, Ryter SW and Levine B: Autophagy in human health and disease. N Engl J Med 368: 651-662, 2013.

5. Chang L and Karin M: Mammalian MAP kinase signalling cascades. Nature 410: 37-40, 2001.

6. Kunath T, Saba-El-Leil MK, Almousailleakh M, Wray J, Meloche $\mathrm{S}$ and Smith A: FGF stimulation of the Erk1/2 signalling cascade triggers transition of pluripotent embryonic stem cells from self-renewal to lineage commitment. Development 134: 2895-2902, 2007.

7. Ge C, Xiao G, Jiang D and Franceschi RT: Critical role of the extracellular signal-regulated kinase-MAPK pathway in osteoblast differentiation and skeletal development. J Cell Biol 176: 709-718, 2007.

8. Wang HY, Yang SL, Liang HF and Li CH: HBx protein promotes oval cell proliferation by up-regulation of cyclin D1 via activation of the MEK/ERK and PI3K/Akt pathways. Int J Mol Sci 15: 3507-3518, 2014.

9. Oh SH, Hatch HM and Petersen BE: Hepatic oval 'stem' cell in liver regeneration. Semin Cell Dev Biol 13: 405-409, 2002.

10. Zhang Q, Yang YJ, Wang H, Dong QT, Wang TJ, Qian HY and $\mathrm{Xu} \mathrm{H}$ : Autophagy activation: A novel mechanism of atorvastatin to protect mesenchymal stem cells from hypoxia and serum deprivation via AMP-activated protein kinase/mammalian target of rapamycin pathway. Stem Cells Dev 21: 1321-1332, 2012. 
11. Couchie D, Holic N, Chobert MN, Corlu A and Laperche Y: In vitro differentiation of WB-F344 rat liver epithelial cells into the biliary lineage. Differentiation 69: 209-215, 2002.

12. She M, Pan I, Sun L and Yeung SC: Enhancement of manumycin A-induced apoptosis by methoxyamine in myeloid leukemia cells. Leukemia 19: 595-602, 2005.

13. Mizushima N and Levine B: Autophagy in mammalian development and differentiation. Nat Cell Biol 12: 823-830, 2010.

14. Phadwal K, Watson AS and Simon AK: Tightrope act: Autophagy in stem cell renewal, differentiation, proliferation, and aging. Cell Mol Life Sci 70: 89-103, 2013.

15. Mizushima N, Levine B, Cuervo AM and Klionsky DJ: Autophagy fights disease through cellular self-digestion. Nature 451: 1069-1075, 2008

16. Guan JL, Simon AK, Prescott M, Menendez JA, Liu F, Wang F, Wang C, Wolvetang E, Vazquez-Martin A and Zhang J: Autophagy in stem cells. Autophagy 9: 830-849, 2013.

17. Zirin J and Perrimon N: Drosophila as a model system to study autophagy. Semin Immunopathol 32: 363-372, 2010.

18. Rubinsztein DC, Gestwicki JE, Murphy LO and Klionsky DJ: Potential therapeutic applications of autophagy. Nat Rev Drug Discov 6: 304-312, 2007.

19. Coller HA, Sang L and Roberts JM: A new description of cellular quiescence. PLoS Biol 4: e83, 2006.

20. Mortensen M, Watson AS and Simon AK: Lack of autophagy in the hematopoietic system leads to loss of hematopoietic stem cell function and dysregulated myeloid proliferation. Autophagy 7 : 1069-1070, 2011.

21. Yang $\mathrm{Z}$ and Klionsky DJ: Eaten alive: A history of macroautophagy. Nat Cell Biol 12: 814-822, 2010.

22. Liu F, Lee JY, Wei H, Tanabe O, Engel JD, Morrison SJ and Guan JL: FIP200 is required for the cell-autonomous maintenance of fetal hematopoietic stem cells. Blood 116: 4806-4814, 2010.

23. Mortensen M, Soilleux EJ, Djordjevic G, Tripp R, Lutteropp M, Sadighi-Akha E, Stranks AJ, Glanville J, Knight S, Jacobsen SE, et al: The autophagy protein Atg7 is essential for hematopoietic stem cell maintenance. J Exp Med 208: 455-467, 2011.

24. Warr MR, Binnewies M, Flach J, Reynaud D, Garg T, Malhotra R, Debnath $\mathrm{J}$ and Passegué E: FOXO3A directs a protective autophagy program in haematopoietic stem cells. Nature 494: 323-327, 2013

25. Oliver L, Hue E, Priault M and Vallette FM: Basal autophagy decreased during the differentiation of human adult mesenchymal stem cells. Stem Cells Dev 21: 2779-2788, 2012.

26. Salemi S, Yousefi S, Constantinescu MA, Fey MF and Simon HU: Autophagy is required for self-renewal and differentiation of adult human stem cells. Cell Res 22: 432-435, 2012.

27. Vázquez P, Arroba AI, Cecconi F, de la Rosa EJ, Boya P and de Pablo F: Atg5 and Ambra1 differentially modulate neurogenesis in neural stem cells. Autophagy 8: 187-199, 2012.

28. Wang S, Li B, Qiao H, Lv X, Liang Q, Shi Z, Xia W, Ji F and Jiao J: Autophagy-related gene Atg5 is essential for astrocyte differentiation in the developing mouse cortex. EMBO Rep 15 $1053-1061,2014$
29. Wang C, Liang CC, Bian ZC, Zhu Y and Guan JL: FIP200 is required for maintenance and differentiation of postnatal neural stem cells. Nat Neurosci 16: 532-542, 2013.

30. Zhang J, Liu J, Huang Y, Chang JY, Liu L, McKeehan WL, Martin JF and Wang F: FRS $2 \alpha$-mediated FGF signals suppress premature differentiation of cardiac stem cells through regulating autophagy activity. Circ Res 110: e29-e39, 2012.

31. Mizushima N, Yamamoto A, Hatano M, Kobayashi Y, Kabeya Y, Suzuki K, Tokuhisa T, Ohsumi Y and Yoshimori T: Dissection of autophagosome formation using Apg5-deficient mouse embryonic stem cells. J Cell Biol 152: 657-668, 2001

32. Tra T, Gong L, Kao LP, Li XL, Grandela C, Devenish RJ, Wolvetang E and Prescott M: Autophagy in human embryonic stem cells. PLoS One 6: e27485, 2011.

33. Cheng Y, Wang B, Zhou H, Dang S, Jin M, Shi Y, Hao L, Yang Z and Zhang Y: Autophagy is required for the maintenance of liver progenitor cell functionality. Cell Physiol Biochem 36: 1163-1174, 2015

34. Heras-Sandoval D, Pérez-Rojas JM, Hernández-Damián J and Pedraza-Chaverri J: The role of PI3K/AKT/mTOR pathway in the modulation of autophagy and the clearance of protein aggregates in neurodegeneration. Cell Signal 26: 2694-2701, 2014

35. Milla LA, González-Ramírez CN and Palma V: Sonic Hedgehog in cancer stem cells: A novel link with autophagy. Biol Res 45: 223-230, 2012

36. Tsao MS, Smith JD, Nelson KG and Grisham JW: A diploid epithelial cell line from normal adult rat liver with phenotypic properties of 'oval' cells. Exp Cell Res 154: 38-52, 1984.

37. Sun C, Jin XL and Xiao JC: Oval cells in hepatitis B virus-positive and hepatitis $\mathrm{C}$ virus-positive liver cirrhosis: Histological and ultrastructural study. Histopathology 48: 546-555, 2006.

38. Zeng J, Jing Y, Shi R, Pan X, Lai F, Liu W, Li R, Gao L, Hou X, Wu M and Wei L: Autophagy regulates biliary differentiation of hepatic progenitor cells through Notch1 signaling pathway. Cell Cycle 15: 1602-1610, 2016.

39. Fukuda T, Oda K, Wada-Hiraike O, Sone K, Inaba K, Ikeda Y, Miyasaka A, Kashiyama T, Tanikawa M, Arimoto T, et al: The anti-malarial chloroquine suppresses proliferation and overcomes cisplatin resistance of endometrial cancer cells via autophagy inhibition. Gynecol Oncol 137: 538-545, 2015.

40. Yuan H, He M, Cheng F, Bai R, da Silva SR, Aguiar RC and Gao SJ: Tenovin-6 inhibits proliferation and survival of diffuse large B-cell lymphoma cells by blocking autophagy. Oncotarget 8: 14912-14924, 2017.

41. Mariño G, Niso-Santano M, Baehrecke EH and Kroemer G: Self-consumption: The interplay of autophagy and apoptosis. Nat Rev Mol Cell Biol 15: 81-94, 2014.

42. Gu J, Hu W, Song ZP, Chen YG, Zhang DD and Wang CQ: Rapamycin inhibits cardiac hypertrophy by promoting autophagy via the MEK/ERK/Beclin-1 pathway. Front Physiol 7: 104, 2016.

43. Martinez-Lopez N, Athonvarangkul D, Mishall P, Sahu S and Singh R: Autophagy proteins regulate ERK phosphorylation. Nat Commun 4: 2799, 2013. 